\title{
Some Recent Developments in Lagrangian Mean Curvature Flows
}

\author{
Mu-Tao Wang*
}

\begin{abstract}
We review some recent results on the mean curvature flows of Lagrangian submanifolds from the perspective of geometric partial differential equations. These include global existence and convergence results, characterizations of first-time singularities, and constructions of self-similar solutions.
\end{abstract}

\section{Introduction}

1.1. The mean curvature flow. A distinguished normal vector field called the mean curvature vector field exists on any submanifold of a Riemannian manifold. It is characterized as the unique direction along which the area or the volume of the submanifold would be decreased most effectively. A submanifold is minimal if the mean curvature vector vanishes at each point. The mean curvature flow is an evolution process that moves a submanifold by its mean curvature vector field. This turns out to be a nonlinear parabolic system of partial differential equations for the position functions of the submanifold. Brakke $[\mathbf{B R}]$ pioneered the formulation of weak solutions of the mean curvature flow in the setting of geometric measure theory in the seventies. In the eighties, Huisken and his coauthors [HU1] took up the geometric PDE approach to study the mean curvature flow in Riemannian manifolds. There were also level set formulations of viscosity solutions for hypersurfaces by Chen-Giga-Goto [CGG] and independently by Evans-Spruck [ES1] later. Soon the mean curvature flow joined the rank of most studied geometric evolution equations like the Ricci flow and the harmonic map heat flows. Indeed, in many aspects, its development paralleled that of the Ricci flow.

Suppose $M$ is a $m$ dimensional Riemannian manifold with a Riemannian metric $\langle\cdot, \cdot\rangle$. Let $\Sigma$ be another $n$ dimensional smooth manifold with $n<m$.

${ }^{*}$ The author is partially supported by National Science Foundation Grant DMS 0605115 and an Alfred P. Sloan Research Fellowship.

(C)2008 International Press 
An immersion of $\Sigma$ in $M$ is given by a mapping $F: \Sigma \rightarrow M$ so that the differential $d F$ has full rank at each point of $\Sigma$. In terms of local coordinates $x^{1} \cdots x^{n}$ on $\Sigma$, this is equivalent to the matrix $g_{i j}=\left\langle\frac{\partial F}{\partial x^{i}}, \frac{\partial F}{\partial x^{j}}\right\rangle$ being positive definite. Indeed, $g_{i j}$ defines a Riemannian metric which makes the image $F(\Sigma)$ a Riemannian submanifold. The second fundamental form of $F(\Sigma)$ is given by the tensor $A=\left(\nabla_{\frac{\partial F}{\partial x^{i}}} \frac{\partial F}{\partial x^{j}}\right)^{\perp}$ where $\nabla$ denotes the Levi-Civita connection of $M$ and $\perp$ denotes the normal part of a vector in the bundle $\left.T M\right|_{F(\Sigma)}$. The mean curvature vector $H$ is then the trace of the second fundamental form, i.e.,

$$
H=g^{i j}\left(\nabla_{\frac{\partial F}{\partial x^{i}}} \frac{\partial F}{\partial x^{j}}\right)^{\perp}
$$

where $g^{i j}$ is the inverse of $g_{i j}$.

We recall the volume of $F(\Sigma)$ is calculated by

$$
\operatorname{Vol}(F(\Sigma))=\int_{\Sigma} \sqrt{\operatorname{det} g_{i j}} d x^{1} \wedge \cdots \wedge d x^{n}
$$

Suppose $F: \Sigma \times[0, \epsilon) \rightarrow M$ is a family of immersion so the variation field $\frac{\partial F}{\partial s}$ is a normal vector field along $F(\Sigma, s)$. The variation of the volume is then given by

$$
\frac{d}{d s} \operatorname{Vol}(F(\Sigma, s))=-\int_{\Sigma}\left\langle\frac{\partial F}{\partial s}, H\right\rangle \sqrt{\operatorname{det} g_{i j}} d x^{1} \wedge \cdots \wedge d x^{n} .
$$

The mean curvature flow deforms a submanifold in the direction of the mean curvature vector field $H$. Namely, a family of immersion $F: \Sigma \times$ $[0, T) \rightarrow M$ is said to form a mean curvature flow if

$$
\left(\frac{\partial F}{\partial t}(x, t)\right)^{\perp}=H(F(x, t)) .
$$

The flow is a nonlinear weakly parabolic system for $F$ and is invariant under reparametrization of $\Sigma$. Indeed, by coupling with a diffeomorphism of $\Sigma$, the flow can be made into a normal deformation, i.e., $\frac{\partial F}{\partial t}(x, t)$ is always in the normal direction. One can establish the short-time existence for any smooth compact initial data. For a normal deformation, a simple calculation shows

$$
\frac{d}{d t} \sqrt{\operatorname{det} g_{i j}(F(x, t))}=-|H(F(x, t))|^{2} \sqrt{\operatorname{det} g_{i j}(F(x, t))},
$$

from which it follows that being a immersion is preserved by the mean curvature flow. Integrating this equality gives

$$
\frac{d}{d t} \operatorname{Vol}(F(\Sigma, t))=-\int_{\Sigma}|H(F(x, t))|^{2} \sqrt{\operatorname{det} g_{i j}(F(x, t))} d x^{1} \wedge \cdots \wedge d x^{n}
$$


In the rest of the paper, when there is no confusion, we will not differentiate between an immersed submanifold $\Sigma$ and its image $F(\Sigma) . T \Sigma$ will denote the tangent bundle and $N \Sigma$ will denote the normal bundle of $\Sigma$. We shall denote the image $F(\Sigma, t)$ of a mean curvature flow by $\Sigma_{t}$ and the volume form $\sqrt{\operatorname{det} g_{i j}(F(x, t))} d x^{1} \wedge \cdots \wedge d x^{n}$ by $d v_{\Sigma_{t}}$.

1.2. Co-dimension one vs. higher co-dimension. The Lagrangian mean curvature flow is a special case of the mean curvature flow in general. They are mostly higher co-dimensional in the sense that $m>n+1$ where $m$ is the dimension of the ambient manifold and $n$ is the dimension of the evolving submanifold. There are abundant results of hypersurface (i.e., $m=n+1$ ) mean curvatures flows, while relatively little is known in the higher co-dimensional case. Indeed, many techniques and results for hypersurface flows do not generalize to higher co-dimensions. The contrast between the hypersurface and higher co-dimensional can be seen from two points. Firstly, the hypersurface case corresponds to a scalar equation and the maximum principle holds in the following sense. Two embedded hypersurfaces evolving by the mean curvature flow will avoid each other and an embedded hypersurface remains embedded along the mean curvature flow. These are no longer true for higher co-dimensional mean curvature flows. Secondly, the second fundamental form of a hypersurface is a symmetric two tensor and various convexity conditions associated with this tensor play an important role. However, for a higher co-dimensional submanifold, the second fundamental is a symmetric two tensor valued in the normal bundle and there is no natural convexity condition for such a tensor. Of course, both these points are related to the complexity of the normal bundle. The normal bundle of an embedded orientable hypersurface is always trivial, while the normal bundle of a higher co-dimensional submanifold could be highly non-trivial.

The good news is that many great results like Brakke's regularity theorem [BR], Hamilton's maximum principle for tensors [HA], Huisken's monotonicity formula $[\mathbf{H U 2}]$, and White's regularity theorem $[\mathbf{W H}]$ are valid in any dimension and co-dimension.

1.3. What is special about being Lagrangian?. A Lagrangian submanifold sits in a symplectic manifold $M$. Recall a symplectic manifold is a smooth manifold equipped with a closed non-degenerate two-form $\omega(\cdot, \cdot)$. A Lagrangian submanifold $\Sigma$ is characterized by the vanishing of $\left.\omega\right|_{\Sigma}$. To get the volume functional into the context, we consider on $M$ a Riemannian metric $\langle\cdot, \cdot\rangle$ and a compatible almost complex structure $J$ in the sense that $\omega(\cdot, \cdot)=\langle J(\cdot), \cdot\rangle$.

The simplest examples are Lagrangian submanifolds in the complex Euclidean space $\mathbb{C}^{n}$. Suppose $z^{i}=x^{i}+y^{i}, i=1 \ldots n$ are complex coordinates on $\mathbb{C}^{n}$. Let $\omega=\sum_{i=1}^{n} d x^{i} \wedge d y^{i}$ be the standard symplectic form on $\mathbb{C}^{n}$ and $J\left(\frac{\partial}{\partial x^{i}}\right)=\frac{\partial}{\partial y^{i}}$ be the standard almost complex structure. We have 
$\omega(X, Y)=\langle J X, Y\rangle$ where $\langle\cdot, \cdot\rangle$ is the standard metric on $\mathbb{C}^{n}$. Recall a Lagrangian subspace of $\mathbb{C}^{n}$ is a subspace on which $\omega$ restricts to zero. As the form $\omega$ is invariant under the unitary group $U(n)$, the set of all Lagrangian subspaces in $\mathbb{C}^{n}$, so called the Lagrangian Grassmannian, is isomorphic to the homogeneous space $U(n) / S O(n)$. Therefore a Lagrangian submanifold is simply a submanifold whose tangent spaces are all Lagrangian subspaces in $\mathbb{C}^{n}$.

Two prominent classes of Lagrangian submanifolds are the following.

1) Graphs of symplectomorphisms: In this case, the ambient space is the product of two symplectic manifolds. Take a smooth map $f:\left(\mathbb{C}^{n}, \omega_{1}\right) \rightarrow\left(\mathbb{C}^{n}, \omega_{2}\right)$ such that $f^{*} \omega_{2}=\omega_{1}$ and the graph of $f$ in $\left(\mathbb{C}^{n} \times \mathbb{C}^{n}, \omega_{1}-\omega_{2}\right)$ is such an example.

2) Graphs of one-forms: In this case, the ambient space is the cotangent bundle of a symplectic manifold. Take a smooth map $f: \mathbb{R}^{n} \rightarrow$ $\mathbb{R}^{n}$ such that $f=\nabla u$ for some scalar function $u: \mathbb{R}^{n} \rightarrow \mathbb{R}$. Identify the first $\mathbb{R}^{n}$ with the real part of $\mathbb{C}^{n}$ with coordinates $x^{i}$ and the second $\mathbb{R}^{n}$ as the imaginary part with coordinates $y^{i}$. The graph of $f$ in $\left(\mathbb{C}^{n}=\mathbb{R}^{n} \oplus \sqrt{-1} \mathbb{R}^{n}, \omega\right)$ with $\omega=\Sigma_{i=1}^{n} d x^{i} \wedge d y^{i}$ is such an example.

Two remarkable properties of Lagrangian mean curvature flow makes one speculate that it perhaps behaves in a better way than other mean curvature flows in higher codimension. Firstly, the normal bundle of a Lagrangian submanifold is canonically isometric to the tangent bundle by the almost complex structure $J: T \Sigma \rightarrow N \Sigma$. This gives a simpler description of the second fundamental form as a fully symmetric three tensor in $\odot^{3} T \Sigma$, the coefficients of which being

$$
h_{i j k}=\left\langle\nabla_{\frac{\partial F}{\partial x^{i}}} \frac{\partial F}{\partial x^{j}}, J\left(\frac{\partial F}{\partial x^{k}}\right)\right\rangle .
$$

As $J H$ becomes a tangent vector, it is dual to a one-form $\sigma$ on $\Sigma$; they are related by $\sigma(\cdot)=\omega(H, \cdot)$.

Secondly, when the ambient space is Kähler-Einstein, being Lagrangian is a condition that is preserved along the mean curvature flow [SM1]. In many occasions, one is tempted to compare the Lagrangian mean curvature flow to the Kähler-Ricci flow. This will be elaborated in the next section when we consider a Calabi-Yau ambient manifold.

1.4. Calabi-Yau case. The Lagrangian mean curvature flow is of particular interest when the ambient space is Calabi-Yau. Let $(M, g, \omega, J)$ be a real $2 n$ dimensional Kähler-Einstein manifold; i.e., the Ricci form is a multiple of the the Kähler form:

$$
\text { Ric }=c \omega .
$$


Let $\Sigma$ be an $n$ dimensional Lagrangian submanifold of $M$. By the Codazzi equation, we have

$$
d \sigma=\left.R i c\right|_{\Sigma}=\left.c \omega\right|_{\Sigma}=0
$$

Thus $\sigma$ is a closed one-form and defines a cohomology class $[\sigma]$.

When $M$ is Calabi-Yau with a canonical parallel holomorphic $(n, 0)$ form $\Omega$. By suitable normalization, the restriction of $\Omega$ on $\Sigma$ gives a multi-valued function $\theta$, called the phase function. Indeed, $*_{\Sigma} \Omega=e^{i \theta}$ where $*_{\Sigma}$ denote the Hodge star operator on $\Sigma$. It turns out the mean curvature form is $\sigma=d \theta$. $[\sigma]$ is called the Maslov class and can be defined through the Gauss map of $\Sigma$.

Lagrangian submanifolds with vanishing mean curvature $H$ are minimal Lagrangian submanifolds. When $M$ is Calabi-Yau, a connected minimal Lagrangian has constant phase and is called a special Lagrangian which corresponds to a class of calibrated submanifolds first studied by Harvey and Lawson $[\mathbf{H L}]$. They also play important roles in the SYZ [SYZ] conjecture in Mirror symmetry. Roughly speaking, people expect special Lagrangians to behave much like holomorphic curves. It is thus desirable to have a general method of constructing special (or minimal) Lagrangian submanifolds. Schoen and Wolfson [SW1] studied the existence problem by the variational method. Another approach more related to algebraic geometry and symplectic topology has been investigated by Joyce.

Based on the duality between graded Lagrangian submanifolds and stable vector bundles, Thomas and Yau $[\mathbf{T Y}]$ made the following conjecture:

Conjecture 1. Let $M$ be Calabi-Yau and $\Sigma$ be a compact embedded Lagrangian submanifold with zero Maslov class, then the mean curvature flow of $\Sigma$ exists for all time and converges smoothly to a special Lagrangian submanifold in the Hamiltonian isotopy class of $\Sigma$.

Suppose $\Sigma_{t}$ is the mean curvature flow of $\Sigma$. The phase function $\theta$ on $\Sigma_{t}$ is evolved by the heat equation

$$
\frac{d \theta}{d t}=\Delta_{\Sigma_{t}} \theta
$$

where $\Delta_{\Sigma_{t}}$ is the Laplace operator of the induced metric on $\Sigma_{t}$. Thus being of zero Maslov class is preserved along the flow. This is a rather bold conjecture as it is easy to see that the mean curvature flow of any compact submanifold of the Euclidean space develops finite time singularities. The most common singularity is the so-called neck-pinching. Without the assumption on the Maslov class, Schoen and Wolfson [SW2] construct example that develop such singularities in finite time. We shall come back to this point in $§ 2.3$.

1.5. Overview of the article. In this paper, we review some recent results on the mean curvature flows of Lagrangian submanifolds. The review 
is by no means comprehensive or complete and is subject to the author's personal preference. Many other interesting works on the Lagrangian mean curvature flow are not discussed in the article, e.g., [GSSZ], [LW], [NE2], and $[\mathbf{P A}]$.

A fundamental question in geometric flows is under what conditions on the initial data can we prove the global existence and convergence in the smooth category. The mean curvature flow, as a quasi-linear system, forms singularity exactly when the second fundamental form of the submanifold blows up, i.e., $\Sigma_{t}$ becomes singular as $t$ approaches $T$ if and only if $\lim _{t \rightarrow T} \sup _{\Sigma_{t}}|A|^{2} \rightarrow \infty$. In $\S 2.1$, we discuss global existence and convergence results for special initial data that correspond to the two classes of Lagrangian submanifolds discussed in $§ 1.3$.

For more general initial data set, we need to understand singularity formations through the blow-up analysis. Suppose the flow exists on $[0, T)$ and $\sup _{\Sigma_{t}}|A|^{2} \rightarrow \infty$ as $t \rightarrow T$, we perform parabolic blow-up of the solution near $T$. For simplicity, here we demonstrate the idea in the case when the ambient space is the Euclidean space $\mathbb{R}^{N}$. This process depends on three parameters $t_{i}$ (when), $y_{i}$ (where), and $\lambda_{i}$ (how much) and eventually we let $\lambda_{i} \rightarrow \infty$. Take the space time track of the mean curvature flow $\mathfrak{M}=\cup_{t \in[0, T)} \Sigma_{t}$ in $\mathbb{R}^{N} \times \mathbb{R}$ and consider the map

$$
\mathbb{R}^{N} \times[0, T) \rightarrow \mathbb{R}^{N} \times\left[-\lambda_{i}^{2} t_{i}, \lambda_{i}^{2}\left(T-t_{i}\right)\right)
$$

by sending $(y, t) \mapsto\left(\lambda_{i}\left(y-y_{i}\right), \lambda_{i}^{2}\left(t-t_{i}\right)\right)$ and thus $\left(y_{i}, t_{i}\right) \mapsto(0,0)$.

The image of $\Sigma_{t}$ can be described as

$$
\Sigma_{s}^{i}=\lambda_{i}\left(\Sigma_{t_{i}+\frac{s}{\lambda_{i}^{2}}}-y_{i}\right)
$$

in which $s=\lambda_{i}^{2}\left(t-t_{i}\right)$. It turns out the space-time track $\mathfrak{M}_{i}=\cup_{s} \Sigma_{s}^{i}$ forms another mean curvature flow by the invariance of the scaling that lives in $\left[-\lambda_{i}^{2} t_{i}, \lambda_{i}^{2}\left(T-t_{i}\right)\right)$.

In order to obtain a smooth limit, two types of parabolic blow-ups are often used depending on how fast the second fundamental form blows up.

Type I blow-up: Also called a central blow-up where the center $\left(t_{i}, y_{i}\right)=$ $\left(T, y_{0}\right)$ is fixed and $\lambda_{i} \rightarrow \infty$. When $|A|^{2}(T-t)$ is bounded, the limit is smooth. Nevertheless, the limit always exists weakly in the sense of geometric measure theory and is an ancient self-similar solution that lives in $(-\infty, 0]$. This is the parabolic analogue of a cone.

Type II blow up: The blow-up center $\left(y_{i}, t_{i}\right)$ is at a point where $|A|^{2}(T-t)$ almost achieves its maximum. The scale is proportional to $|A|^{2}$ so we get a smooth limit that often lives on $(-\infty, \infty)$ with uniformly bounded second fundamental form, so called an eternal solution. In $\S 2.2$, we discuss the characterization of first time singularity for Lagrangian mean curvature flow under the Type I blow-up procedure. 
The singularities are often classified into type I singularity or type II singularity according to whether $\sup _{\Sigma_{t}}|A|^{2}(T-t)$ is bounded or unbounded as $t \rightarrow T$ (not to be confused with the type I and type II blow-up procedures). The simplest Lagrangian mean curvature flow reduces to the socalled curve shortening flow on a two-dimensional orientable surface as any curve is Lagrangian. In this case, it can be proved that any type I singularity is a shrinking circle and any type II singularity is a Grim Reaper, both are self-similar solutions. It is thus concievable that the eventual understandings of the singularities will rely on the classifications of self-similar solutions. In $§ 2.3$, we discuss the constructions of self-similar solutions in Lagrangian mean curvature flows.

The author would like to thank B. Andrews, K. Ecker, R. Hamilton, M. Haskin, G. Huisken, T. Ilmanen, D. Joyce, Y.-I. Lee, N. C. Leung, A. Neves, K. Smoczyk, M.-P. Tsui, T. Y. H. Wan, and B. White for helpful discussions on this subject.

\section{Results}

2.1. Global existence and convergence. Given an immersed submanifold $\Sigma_{0}$ of a Riemannian manifold $M$, we ask when we can find a family of immersions that forms a mean curvature flow $\Sigma_{t}$ and when $\Sigma_{t} \rightarrow$ $\Sigma_{\infty}$ in $C^{\infty}$ for a smooth immersed submanifold $\Sigma_{\infty}$. As was remarked in the overview of the article, this boils down to bounding the second fundamental form for all $t \in[0, \infty)$ and as $t \rightarrow \infty$. The one-dimensional curve-shortening flow is a well-studied area and there are many beautiful global existence and convergence results by, e.g., Gage-Hamilton $[\mathbf{G H}]$ and Grayson[GR1][GR2]. We refer to the book by Chou-Zhu $[\mathbf{C Z}]$ for results in this direction.

The next simplest case will be two-dimensional Lagrangian surfaces in a four-dimensional symplectic manifold. We recall that the graph of a symplectomorphism is naturally a Lagrangian submanifold of the product space. In this case, there is the following theorem:

ThEOREM 2.1. Let $\left(\Sigma^{(1)}, \omega_{1}\right)$ and $\left(\Sigma^{(2)}, \omega_{2}\right)$ be two diffeomorphic compact Riemann surfaces of the same constant curvature c. Suppose $\Sigma$ is the graph of a symplectomorphism $f: \Sigma^{(1)} \rightarrow \Sigma^{(2)}$ as a Lagrangian submanifold of $M=\left(\Sigma^{(1)} \times \Sigma^{(2)}, \omega=\omega_{1}-\omega_{2}\right)$ and $\Sigma_{t}$ is the mean curvature flow with initial surface $\Sigma_{0}=\Sigma$. Then $\Sigma_{t}$ remains the graph of a symplectomorphism $f_{t}$ along the mean curvature flow. The flow exists smoothly for all time and $\Sigma_{t}$ converges smoothly to a minimal Lagrangian submanifold as $t \rightarrow \infty$.

The assumption on the curvatures of $\Sigma^{(1)}$ and $\Sigma^{(2)}$ makes $M$ a KählerEinstein manifold with the product metric. The long time existence for all cases and the smooth convergence for $c>0$ was proved in [WA2]. The smooth convergence for $c \leq 0$ was established in [WA4]. 
Assuming an extra angle condition, Smoczyk [SM2] proved the theorem when $c \leq 0$. Smoczyk's proof of the global existence and convergence result is different from that of [WA2] and [WA4]. Instead of applying blow-up analysis, he proved directly by the maximum principle that the second fundamental form is uniformly bounded independent of time. This gives the global existence and the convergence at the same time.

We remark the existence of such minimal Lagrangian submanifold was proved using variational method by Schoen $[\mathbf{S C}]$ (see also Lee $[\mathbf{L E}]$ ).

In the following, we briefly describe the proof of the theorem. The proof is divided into three parts.

1) $\Sigma_{t}$ remains the graph of a symplectomorphism $f_{t}$ as long as the flow exists smoothly.

Since $M$ is Kähler-Einstein, $\Sigma_{t}$ remains a Lagrangian surface. This can indeed be shown by considering the evolution equation satisfied by the function $* \omega(p)=\omega\left(e_{1}, e_{2}\right)$ where $\left\{e_{1}, e_{2}\right\}$ is any oriented orthonormal basis for $T_{p} \Sigma$. Likewise, we can consider the heat equation satisfied by the function $* \omega_{1}(p)=\omega_{1}\left(e_{1}, e_{2}\right)$. In an orthonormal basis, we can represent the second fundamental form $A$ by $h_{i j k}=\left\langle\nabla_{e_{i}} e_{j}, J\left(e_{k}\right)\right\rangle$ and the mean curvature vector by $H_{k}=\left\langle H, J\left(e_{k}\right)\right\rangle=\sum_{i=1}^{2} h_{i i k}$. It was computed in [WA2] that $\eta_{t}=2 * \Sigma_{t} \omega_{1}$ satisfies

$$
\frac{d}{d t} \eta_{t}=\Delta \eta_{t}+\eta_{t}\left(2|A|^{2}-|H|^{2}\right)+c \eta_{t}\left(1-\eta_{t}^{2}\right)
$$

where $|A|^{2}=\sum_{i, j, k} h_{i j k}^{2}$ and $|H|^{2}=\sum_{k} H_{k}^{2}$ are the squared norms of the second fundamental form and the mean curvature vector, respectively. As $|H|^{2} \leq 2|A|^{2}, \eta_{t}>0$ is preserved along the flow by the maximum principle.

Notice that $* \omega_{1}$ is in fact the Jacobian of $\left.\pi_{1}\right|_{\Sigma}$ where $\pi_{1}: \Sigma^{(1)} \times \Sigma^{(2)} \rightarrow$ $\Sigma^{(1)}$ is the projection map onto the first factor. By the inverse function theorem, $* \eta_{t}>0$ if and only if $\Sigma_{t}$ can be locally written a graph over $\Sigma_{1}$. Therefore $\eta_{t}>0$ implies $\Sigma_{t}$ is the graph of a symplectomorphism.

Indeed, not only $\eta_{t}>0$ but by comparing to solutions of the ordinary differential equation $\frac{d}{d t} f=c f\left(1-f^{2}\right)$, we arrive at

$$
\eta_{t} \geq \frac{\alpha e^{c} t}{\sqrt{1+\alpha^{2} e^{2 c t}}}
$$

where $\alpha$ is a constant that satisfies $\frac{\alpha}{\sqrt{1+\alpha^{2}}}=\min _{\Sigma_{0}} \eta$. In particular, $\eta_{t} \rightarrow 1$ as $t \rightarrow \infty$ uniformly when $c>0$.

2) Global existence for all finite time

Utilizing the full symmetry of the second fundamental form $h_{i j k}$, one can show that

Therefore,

$$
|H|^{2} \leq \frac{4}{3}|A|^{2}
$$

$$
\frac{d}{d t} \eta_{t} \geq \Delta \eta_{t}+\frac{2}{3} \eta_{t}|A|^{2}+c \eta_{t}\left(1-\eta_{t}^{2}\right)
$$


We can apply the type I blow-up procedure to this solution at any spacetime point. Equation 2.2 and the positive lower bound of $\eta_{t}$ at any finite time will imply the integral of $|A|^{2}$ vanishes on the type-I blow-up limit. It follows from White's regularity theorem $[\mathbf{W H}]$ that any such point is a regular point.

3) Convergence at $t=\infty$.

The aim is to bound $|A|^{2}$ as $t \rightarrow \infty$ since Simon's [SI] convergence theorem for gradient flows is applicable in this case. Suppose $\sup _{\Sigma_{t}}|A|^{2} \rightarrow$ $\infty$, we apply the type II blow-up procedure to the solution at $t=\infty$. Pick a sequence of $t_{i}$ and point $p_{i} \in \Sigma_{t_{i}}$ such that the space-time track $\mathfrak{M}_{i}$, after shifting $\left(p_{i}, t_{i}\right)$ to $(0,0)$ and scaling by the factor $|A|\left(p_{i}, t_{i}\right)$, has uniformly bounded second fundamental form and $|A|(0,0)=1$.

By compactness, $\mathfrak{M}_{i} \rightarrow \mathfrak{M}_{\infty}$, which is an eternal solution of the mean curvature flow defined on $(-\infty, \infty)$ with uniformly bounded second fundamental form and $|A|(0,0)=1$. When $c>0$, recall $\eta_{t} \rightarrow 1$ as $t \rightarrow \infty$, this implies $\eta \equiv 1$ on the limit $\mathfrak{M}_{\infty}$ and each time slice must be a flat space, contradicting with $|A|(0,0)=1$.

In cases when $c \leq 0, \eta_{t}$ no longer converges to 1 as $t \rightarrow \infty$ we consider instead the evolution equation for $|H|^{2}$. It was computed in [WA4] that

$$
\left(\frac{d}{d t}-\Delta\right)|H|^{2}=-2|\nabla H|^{2}+2 \sum_{i j}\left(\sum_{k} H_{k} h_{k i j}\right)^{2}+c\left(2-\eta^{2}\right)|H|^{2} .
$$

Coupling with the equation for $\eta(2.1)$ and integrating over $\Sigma_{t}$ gives

$$
\frac{d}{d t} \int_{\Sigma_{t}} \frac{|H|^{2}}{\eta} d v_{\Sigma_{t}} \leq c \int_{\Sigma_{t}} \frac{|H|^{2}}{\eta} d v_{\Sigma_{t}}
$$

This implies $\int_{\Sigma_{t}}|H|^{2} d v_{\Sigma_{t}} \rightarrow 0$ as $t \rightarrow \infty$ since $\int_{0}^{\infty} \int_{\Sigma_{t}}|H| d v_{\Sigma_{t}} d t<\infty$. The limit $\mathfrak{M}_{\infty}$ obtained earlier has $\int|H|^{2}=0$ and thus each time slice is a minimal area preserving map from $\mathbb{C}$ to $\mathbb{C}$ which must be flat by a result of $\mathrm{Ni}[\mathbf{N I}]$.

In general dimension, Smoczyk and the author $[\mathbf{S W}]$ proved a general global existence and convergence theorem for Lagrangian graphs in $T^{2 n}$, a flat torus of dimension $2 n$.

THEOREM 2.2. Let $\Sigma$ be a Lagrangian submanifold in $T^{2 n}$. Suppose $\Sigma$ is the graph of $f: T^{n} \rightarrow T^{n}$ and the potential function $u$ of $f$ is convex. Then the mean curvature flow of $\Sigma$ exists for all time, remains a Lagrangian graph, and converges smoothly to a flat Lagrangian submanifold.

The flow in terms of the potential $u$ is a fully nonlinear parabolic equation:

$$
\frac{d u}{d t}=\frac{1}{\sqrt{-1}} \ln \frac{\operatorname{det}\left(I+\sqrt{-1} D^{2} u\right)}{\sqrt{\operatorname{det}\left(I+\left(D^{2} u\right)^{2}\right)}}
$$


where $D^{2} u$ is the Hessian of $u$. Notice $\frac{\operatorname{det}\left(I+\sqrt{-1} D^{2} u\right)}{\sqrt{\operatorname{det}\left(I+\left(D^{2} u\right)^{2}\right)}}$ is a unit complex number, so the right hand side is always real. This theorem generalizes prior global existence and convergence results in general dimensions in [WA3] and [SM3].

A important step in the proof is to show the convexity condition $D_{i j}^{2} u>0$ is preserved which we describe in the rest of this section. This involves interpreting the convexity condition as the positivity of some symmetric two tensor on $\Sigma_{t}$ and compute the parabolic equation with respect to the induced (evolving metric) on $\Sigma_{t}$. It turns out if we denote $\pi_{1}\left(\pi_{2}\right)$ to be the projection onto the first (second) factor of $T^{n} \times T^{n}$. The condition $D_{i j}^{2} u$ is the same as

$$
\left\langle J \pi_{1}(X), \pi_{2}(X)\right\rangle>0
$$

for any $X \in T \Sigma . S(\cdot, \cdot)=\left\langle J \pi_{1}(\cdot), \pi_{2}(\cdot)\right\rangle$ defines a two-tensor on $T^{2 n}$ and the Lagrangian condition implies the restriction of $S$ to any Lagrangian submanifold is a symmetric tensor.

LEMMA 2.1. This positivity of $S$ is preserved along the mean curvature flow, i.e., $\left.S\right|_{\Sigma_{t}}>0$ for $t>0$ as long as $\left.S\right|_{\Sigma_{0}}>0$.

A direct approach is to calculate the evolution of $\left.S\right|_{\Sigma_{t}}$ and apply Hamilton's maximal principle for tensors, see equation (3.3) in [SW].

Another more systematic approach is to study how the tangent space of $\Sigma_{t}$ evolves as this contains the information of $D_{i j}^{2} u$. Since the tangent space of $T^{2 n}$ can be identified with $\mathbb{C}^{n}$. We may consider the Gauss map of $\Sigma_{t}$ given by $\gamma_{t}: \Sigma_{t} \rightarrow L G(n)=U(n) / S O(n)$, the Lagrangian Grassmannian, by sending a point $p \in \Sigma$ to the tangent space $T_{p} \Sigma \subset \mathbb{C}^{n}$. The following theorem is proven in [WA5]

THEOREM 2.3. $\gamma_{t}$ is a harmonic map heat flow.

For an ordinary heat equation $\frac{d}{d t} f=\Delta f$, the conditions $f>0$ and $f=0$ are preserved by the maximum principle. For a harmonic map heat flow into a Riemannian manifold, the analogy is that the image of the map will remain in a convex or totally geodesic set.

Since $L G(n)$ is totally geodesic subset of the Grassmannian, this provides an alternate way to show why being Lagrangian is preserved along the mean curvature flow. Also the determinant map from $U(n) / S O(n)$ to $U(1) \equiv S^{1}$ is totally geodesic. Thus the composition $\gamma_{t}$ o det is a harmonic map heat flow into $S^{1}$. It is easy to see that $\gamma_{t} \circ$ det is exactly the phase function $\theta$ and this is another way to derive (1.2).

To show $\left\{L \in L G(n),\left.S\right|_{L}>0\right\}$ is a convex subset, we study the geodesic equation on $L G(n)$ and the details can be found in $[\mathbf{S W}]$. We remark that as being a minimal Lagrangian in $\mathbb{C}^{n}$ is an invariant property under the symmetry group $U(n)$. The equation of $u$ indeed enjoys more symmetric 
than a general fully non-linear Hessian equation. This observation provides more equivalent conditions under which the global existence and convergence theorems can be proved (see the last section in $[\mathbf{S W}]$ ).

2.2. Characterization of first-time singularities. In [WA1], the author introduced the notion of almost calibrated Lagrangian submanifolds in the study of characterizing the first time singularity. Recall for a special Lagrangian, the Lagrangian angle (after a shifting) satisfies $\cos \theta=1$. A Lagrangian submanifold in a Calabi-Yau manifold is said to be "almost calibrated" if $\cos \theta \geq \epsilon$ for some $\epsilon>0$. This has proved to be a very useful condition in the study of Lagrangian mean curvature flow.

As $\cos \theta$ satisfies

$$
\frac{d}{d t} \cos \theta=\Delta \cos \theta+\cos \theta|H|^{2}
$$

being almost calibrated is another condition that is preserved along the Lagrangian mean curvature flow. The following theorem is proved in [WA1].

THEOREM 2.4. An almost calibrated Lagrangian submanifold does not develop any type I singularity along the mean curvature flow.

This is established by coupling equation (2.4) with Huisken's monotonicity formula. In particular, no "neck-pinching" will be forming in the Thomas-Yau conjecture if this condition is assumed. To demonstrate the idea, let us pretend the Lagrangian submanifolds are compact and lie in $\mathbb{R}^{n}$. As long as characterizing finite time singularity is concerned, this does not pose any serious restriction as the ambient curvature will be scaled away under a blow-up procedure. Very mild assumption needs to be imposed at infinity to assure the integration by parts work. Suppose the flow exists on $[0, \infty)$ and consider the backward heat kernel at $\left(y_{0}, T\right)$.

$$
\rho_{y_{0}, T}(y, t)=\frac{1}{(4 \pi(T-t))^{\frac{n}{2}}} \exp \left(\frac{-\left|y-y_{0}\right|^{2}}{4(T-t)}\right)
$$

Huisken's monotonicity formula implies

$$
\frac{d}{d t} \int_{\Sigma_{t}} \rho_{y_{0}, T} d v_{\Sigma_{t}}=-\int_{\Sigma_{t}}\left|H+\frac{1}{2(T-t)} F^{\perp}\right|^{2} \rho_{y_{0}, T} d v_{\Sigma_{t}} .
$$

Coupling with the equation for $\cos \theta(2.4)$, we obtain

$$
\begin{aligned}
\frac{d}{d t} \int_{\Sigma_{t}} \rho_{y_{0}, T}(1-\cos \theta) d v_{\Sigma_{t}}= & -\int_{\Sigma_{t}} \rho_{y_{0}, T}|H|^{2} \cos \theta d v_{\Sigma_{t}} \\
& -\int_{\Sigma_{t}}\left|H+\frac{1}{2(T-t)} F^{\perp}\right|^{2}(1-\cos \theta) d v_{\Sigma_{t}} .
\end{aligned}
$$


Both these equations are scaling invariants and continue to hold for the type I blow-up at $\left(y_{0}, T\right)$ (notice that $\theta$ is a scaling invariant) and thus

$$
\frac{d}{d s} \int_{\Sigma_{s}^{i}} \rho(1-\cos \theta)=-\int_{\Sigma_{s}^{i}} \rho|H|^{2} \cos \theta-\int_{\Sigma_{s}^{i}} \rho\left|H-\frac{1}{2 s} F^{\perp}\right|^{2}(1-\cos \theta)
$$

where $\rho$ is the backward heat kernel at $(0,0)$ and $s=-\lambda_{i}^{2}(T-t)$. Therefore it is not hard to see that there exists a sequence of rescaled submanifolds on which both $\int|H|^{2}$ and $\int\left|H-\frac{1}{2 s} F^{\perp}\right|^{2}$ are approaching zero. Thus $H=0$ and $F^{\perp}=0$ weakly on each time slice of the limit. This indicates that each time slice of the limit should be a union of minimal Lagrangian cones. If we assume the singularity is of type I, then the limit flow is smooth and thus must be a flat space. White's regularity theorem implies the point is a smooth point.

Notice that (2.6) implies local area bound and (2.7) implies local bound for the $L^{2}$ norm of mean curvatures on $\Sigma_{s}^{i}$. It follows from compactness theorems in geometric measure theory that the limit is rectifiable and this was carried out by Chen and $\mathrm{Li}$ in $[\mathbf{C L}]$. As $|H|^{2}=|\nabla \theta|^{2}$, a natural question arises whether the phase $\theta$ is a constant on this union of minimal Lagrangian cones. Notice that even a union of special Lagrangian cones may have different phases and hence not necessarily area-minimizing. Chen and $\mathrm{Li}[\mathbf{C L}]$ claimed that the phase function is a constant on the limit by proving a Poincáre inequality for $\theta$. Unfortunately, the proof of Theorem 5.1 in $[\mathbf{C L}]$ overlooks some technical difficulties. Neves later gives a different proof assuming two extra conditions and using the evolution equation of the Liouville form $\lambda=\sum_{i=1}^{n} x^{i} d y^{i}-y^{i} d x^{i}$. We refer to his paper [NE1] for the precise statement of the theorem (Theorem B). Neves [NE1] was also able to replace the assumption of almost calibrated by zero Maslov class by observing that the equation for $\cos \theta \operatorname{can}$ be replaced by

$$
\frac{\partial}{\partial t} \theta^{2}=\Delta \theta^{2}-2|H|^{2}
$$

2.3. Constructions of self-similar solutions. A important tool in the study of geometric flows is the blow-up analysis. A blow-up solution of the mean curvature flow sits in the Euclidean space and often enjoys more symmetry. It is important to study these special solutions as singularity models. A mean curvature flow in the Euclidean space is said to be selfsimilar if it is moved by an ambient symmetry. We may consider ansatz of the type

$$
F(x, t)=\phi(t) F(x)
$$

and

$$
F(x, t)=F(x)+\psi(t)
$$


which correspond to scaling symmetry and translating symmetry, respectively. The ansatz coupled with the mean curvature flow equation gives an elliptic equation for $F(x)$. For a solution of the form $(2.8), F(x, t)$ is called an expanding or a shrinking soliton depending on whether $\phi(t)$ is greater or less than one, respectively. A mean curvature flow $F(x, t)$ that satisfies $(2.9)$ is called a translating soliton.

Henri Anciaux constructed examples of Lagrangian shrinking and expanding solitons in $[\mathbf{A N}]$. All the examples are based on minimal Legendrian immersions in $S^{2 n-1}$ and the solutions are asymptotic to the associated minimal Lagrangian cones.

Yng-Ing Lee and the author [LWA] constructed examples of self-similar shrinking and expanding Lagrangian mean curvature flows that are asymptotic to Hamiltonian stationary cones. They were able to glue them together to form weak solutions of the mean curvature flow in the sense of Brakke. In a new preprint of Joyce, Lee and Tsui [JLT] constructed new examples of self-similar solutions, in particular translating solitons. [NT] gave some characterizations of translating solitons in the two dimensional case.

\section{Prospects}

There have been several attempts to find counterexamples of the Thomas-Yau conjecture. Other than the examples of Schoen and Wolfson in [SW2], Neves [NE1] constructed almost calibrated complete non-compact Lagrangian surfaces in $\mathbb{C}^{2}$ that develop finite time singularities. However, there is still no genuine counterexample to the Thomas-Yau conjecture as it was stated.

It should be noted that Schoen and Wolfson [SW3] proved the following existence result of special Lagrangians in a K-3 surface.

TheOrem 3.1. Let $X$ be a K3 surface with a Calabi-Yau metric. Suppose that $\gamma \in H_{2}(X ; \mathbb{Z})$ is a Lagrangian class that can be represented by an embedded Lagrangian surface. Then $\gamma$ can be represented by a special Lagrangian surface.

A mean curvature flow proof of this theorem will confirm the ThomasYau conjecture in two dimension. Since it was already shown that there is no type-I singularity, we need to focus on type II singularities in the zero Maslov class or almost calibrated case. A general type-II singularity can be scaled to get an eternal solution with uniformly bounded second fundamental form that exists on $(-\infty, \infty)$. Such a solution of a parabolic equation should be rather special and we hope to say more about it in the near future.

For a general initial data, one is tempted to speculate that, just as in the Ricci flow case, surgeries are necessary in order to continue the flow. It was commented in Perelman's paper $[\mathbf{P E}]$ that when the surgery scale goes to zero, the solution with surgeries should converge to a "weak solution" of the Ricci flow, a notion that has yet to established. Weak formulations for the mean curvature flow are available. However, as weak solutions are 
no longer unique, it is necessary to instruct the flow how to continue after singuarities. The examples found in Lee-Wang [LWA] and Joyce-Lee-Tsui [JLT] start out as shrinking solitons as $t<0$, approach to Schoen-Wolfson cones as $t \rightarrow 0$ and resolve to expanding solitons for $t>0$. They altogether form a Brakke flow.

\section{References}

[AN] H. Anciaux, Construction of Lagrangian self-similar solutions to the mean curvature flow in $\mathbb{C}^{n}$. Geom. Dedicata 120 (2006), 37-48.

[BR] K. A. Brakke, The motion of a surface by its mean curvature. Mathematical Notes, 20. Princeton University Press, Princeton, N.J., 1978.

[CGG] Y. G. Chen, Y. Giga, and Y. Goto, Uniqueness and existence of viscosity solutions of generalized mean curvature flow equations. J. Differential Geom. 33 (1991), no. 3, $749-786$.

[CL] J. Chen and J. Li, Singularity of mean curvature flow of Lagrangian submanifolds. Invent. Math. 156 (2004), no. 1, 25-51.

[CZ] K.-S. Chou and X.-P. Zhu, The curve shortening problem. Chapman \& Hall/CRC, Boca Raton, FL, 2001.

[EH] K. Ecker and G. Huisken, Mean curvature evolution of entire graphs. Ann. of Math. (2) 130 (1989), no. 3, 453-471.

[EH2] K. Ecker and G. Huisken, Interior estimates for hypersurfaces moving by mean curvature. Invent. Math. 105 (1991), no. 3, 547-569.

[ES1] L. C. Evans and J. Spruck, Motion of level sets by mean curvature. I. J. Differential Geom. 33 (1991), no. 3, 635-681.

[GH] M. Gage and R. Hamilton, The heat equation shrinking convex plane curves. J. Differential Geom. 23 (1986), no. 1, 69-96.

[GR1] M. Grayson, The heat equation shrinks embedded plane curves to round points. J. Differential Geom. 26 (1987), no. 2, 285-314.

[GR2] M. Grayson, Shortening embedded curves. Ann. of Math. (2) 129 (1989), no. 1, $71-111$.

[GSSZ] K. Groh, M. Schwarz, K. Smoczyk, and K. Zehmisch, Mean curvature flow of monotone Lagrangian submanifolds. Math. Z. 257 (2007), no. 2, 295-327.

[HA] R. S. Hamilton, Four-manifolds with positive curvature operator. J. Differential Geom. 24 (1986), no. 2, 153-179.

[HA3] R. S. Hamilton, Harnack estimate for the mean curvature flow. J. Differential Geom. 41 (1995), no. 1, 215-226.

[HL] R. Harvey and H. B. Lawson, Calibrated geometries. Acta Math. 148 (1982), 47-157.

[HU1] G. Huisken, Flow by mean curvature of convex surfaces into spheres. J. Differential Geom. 20 (1984), no. 1, 237-266.

[HU2] G. Huisken, Asymptotic behavior for singularities of the mean curvature flow. J. Differential Geom. 31 (1990), no. 1, 285-299.

[JO] D. Joyce, Lectures on special Lagrangian geometry. Global theory of minimal surfaces, 667-695, Clay Math. Proc., 2, Amer. Math. Soc., Providence, RI, 2005.

[JLT] D. Joyce, Y.-I. Lee, and M.-P. Tsui, Self-similar solutions and translating solitons for Lagrangian mean curvature flow. preprint 2007.

[LW] N. C. Leung and T. Y. H. Wan, Hyper-Lagrangian submanifolds of hyperkhler manifolds and mean curvature flow. J. Geom. Anal. 17 (2007), no. 2, 343-364.

[LE] Y.-I. Lee, Lagrangian minimal surfaces in Kähler-Einstein surfaces of negative scalar curvature. Comm. Anal. Geom. 2 (1994), no. 4, 579-592.

[LWA] Y.-I. Lee and M.-T. Wang, Hamiltonian stationary self-shrinkers and selfexpanders of Lagranian mean curvature flows. preprint, arXiv:0707.0239. 
[NE1] A. Neves, Singularities of Lagrangian mean curvature flow: zero-Maslov class case. Invent. Math. 168 (2007), no. 3, 449-484.

[NE2] A. Neves, Singularities of Lagrangian mean curvature flow: monotone case. preprint, arXiv:math/0608401.

[NT] A. Neves and G. Tian, Translating solutions to Lagrangian mean curvature flow. preprint, arXiv:0711.4341.

[NI] L. Ni, A Bernstein type theorem for minimal volume preserving maps. Proc. Amer. Math. Soc. 130 (2002), no. 4, 1207-1210.

[PA] T. Pacini, Mean curvature flow, orbits, moment maps. Trans. Amer. Math. Soc. 355 (2003), no. 8, 3343-3357.

[PE] G. Perelman, Ricci flow with surgery on three-manifolds. arXiv:math/0303109v1.

[SC] R. Schoen, The role of harmonic mappings in rigidity and deformation problems. Complex geometry (Osaka, 1990), 179-200, Lecture Notes in Pure and Appl. Math., 143, Dekker, New York, 1993.

[SW1] R. Schoen and J. Wolfson, Minimizing area among Lagrangian surfaces: the mapping problem. J. Differential Geom. 58 (2001), no. 1, 1-86.

[SW2] R. Schoen and J. Wolfson, Mean curvature flow and lagrangian embeddings. preprint, 2002.

[SW3] R. Schoen and J. Wolfson, in preparation.

[SI] L. Simon, Asymptotics for a class of nonlinear evolution equations, with applications to geometric problems. Ann. of Math. (2) 118 (1983), no. 3, 525-571.

[SM1] K. Smoczyk, A canonical way to deform a Lagrangian submanifold. preprint, dg-ga/9605005.

[SM2] K. Smoczyk, Angle theorems for the Lagrangian mean curvature flow. Math. Z. 240 (2002), no. 4, 849-883.

[SM3] K. Smoczyk, Longtime existence of the Lagrangian mean curvature flow. Calc. Var. Partial Differential Equations 20 (2004), no. 1, 25-46.

[SW] K. Smoczyk and M.-T. Wang, Mean curvature flows of Lagrangians submanifolds with convex potentials. J. Differential Geom. 62 (2002), no. 2, 243-257.

[SYZ] A. Strominger, S.-T. Yau, and E. Zaslow, Mirror symmetry is T-duality. Nuclear Phys. B 479 (1996), no. 1-2, 243-259.

[TY] R. P. Thomas and S.-T. Yau, Special Lagrangians, stable bundles and mean curvature flow. Comm. Anal. Geom. 10 (2002), no. 5, 1075-1113.

[WA1] M.-T. Wang, Mean Curvature Flow of surfaces in Einstein Four-Manifolds. J. Differential Geom. 57 (2001), no. 2, 301-338.

[WA2] M.-T. Wang, Deforming area preserving diffeomorphism of surfaces by mean curvature flow. Math. Res. Lett. 8 (2001), no. 5-6, 651-662.

[WA3] M.-T. Wang, Long-time existence and convergence of graphic mean curvature flow in arbitrary codimension. Invent. Math. 148 (2002), no. 3, 525-543.

[WA4] M.-T. Wang, A convergence result of the Lagrangian mean curvature flow. to appear in the Proceedings of the third International Congress of Chinese Mathematicians. arXiv:math/0508354.

[WA5] M.-T. Wang, Gauss maps of the mean curvature flow. Math. Res. Lett. 10 (2003), no. $2-3,287-299$.

[WH] B. White, A local regularity theorem for classical mean curvature flow. Ann. of Math. (2) 161 (2005), no. 3, 1487-1519.

January 6, 2008

E-mail address: mtwang@math.columbia.edu 
